\section{Construcción del movimiento social en salud en Colombia, 1998-2020}

\section{Building the social health movement in Colombia, 1998-2020}

\section{Construção do movimento social em saúde na Colômbia, 1998-2020}

Yadira Borrero-Ramirez 1

Mauricio Torres-Tovar 2

Maria Esperanza Echeverry-López 1

doi: $10.1590 / 0102-311 \times 00345220$

\section{Resumen}

Esta investigación fue un estudio de caso nacional, Colombia, entre 19982020; que, a partir de análisis documental, entrevistas y observación participante, describe el complejo proceso de configuración de un movimiento social que lucha por la salud. Teóricamente es una perspectiva relacional, que articuló el enfoque de la contienda política y la mirada constructivista, para comprender la constitución del movimiento social por la salud y su relación con los procesos de configuración de la política sanitaria. El artículo se estructura a partir de contextualizar la consolidación del modelo de desarrollo neoliberal y la reforma estructural en salud, en medio de un conflicto armado interno $y$ la contienda politica que se establece entre sectores defensores de la reforma, frente a quienes rechazan y demandan un cambio que garantice el derecho a la salud. A partir de ahí, se identifican y analizan cinco periodos de contienda, cada uno de ellos caracterizado por transformaciones que profundizaban el modelo de mercado en salud, con reconfiguración de actores, repertorios de acción y tensiones internas que permanecen en el tiempo. Se concluye que la identidad de este actor colectivo se ha construido en torno a la salud como derecho humano fundamental y que, dada la heterogeneidad de actores y demandas, ha sido difícil consolidar una plataforma de lucha articulada que logre revertir las reformas de mercado. Además, dado el vínculo de la salud con los determinantes, parece no ser suficiente demandar sistemas universales de salud y se requiere una ampliación de la plataforma de lucha.

Derecho a la Salud; Participación de la Comunidad; Participación Social

\author{
Correspondencia \\ Y. Borrero-Ramirez \\ Facultad Nacional de Salud Pública, Universidad de Antioquia. \\ Calle 55A, 64 BB 36, Medellín, Antioquia 050034, Colombia. \\ yadira.borrero@udea.edu.co \\ 1 Facultad Nacional de Salud Pública, Universidad de \\ Antioquia, Medellín, Colombia. \\ 2 Departamento de Salud Pública, Universidad Nacional de \\ Colombia, Bogotá, Colombia.
}




\section{Introducción}

El campo sanitario colombiano intensifica su conflictividad desde los 1990, cuando empezó su transformación, del anterior Sistema Nacional de Salud al Sistema General de Seguridad Social en Salud (SGSSS) 1,2,3,4,5, caracterizado por: creación de un mercado sanitario, focalización de subsidio a la demanda, transferencia de costos a ciudadanos vía copagos, y aseguramiento individual manejado por intermediarios. Esta configuración generó una contienda pública, con participación de actores colectivos y permanente reconfiguración del movimiento social por la salud 4,5.

Paralelo a la discusión nacional de la reforma, muchos trabajos evidencian la persistencia de inequidades sociosanitarias y la no garantía del derecho a la salud 6,7, demostrando que el SGSSS reproduce inequidades estructurales del país. Este contexto genera malestar social, que deviene en un plural tejido organizativo, amplio debate público, y contiendas legislativas periódicas, que propician la constitución del movimiento social por la salud.

La pandemia de COVID-19 puso nuevamente sobre el escenario las inequidades del SGSSS, así como su capacidad de respuesta. Colombia está entre los 10 primeros países del mundo con mayor número de personas diagnosticadas. Los datos evidencian desigualdades en la mortalidad entre grupos socioeconómicos: $90 \%$ de fallecidos corresponden a estratos socioeconómicos bajos, mientras el 10\% corresponden a los estratos altos 8; los casos mortales superan los 35.000, luego de 9 meses de inicio de la pandemia en Colombia ${ }^{9}$. El manejo gubernamental ha generado un amplio debate desde actores del movimiento social por la salud, así como reactivación de la lucha por el derecho a la salud .

Este trabajo propone analizar el vínculo entre las reformas del Estado, de la política sanitaria, y las etapas atravesadas por el movimiento social por la salud en Colombia, buscando un diálogo entre transformaciones estructurales, configuración de actores colectivos y contiendas disputadas, las cuales se despliegan a través de confrontación conflictiva con la institucionalidad, más que incidencia en la política sectorial, pese a la participación en la contienda legislativa.

Teóricamente, esta investigación considera el movimiento social 10 como un actor sociopolítico, que despliega acciones colectivas, orientadas a enfrentar condiciones de injusticia y son propositivas en contextos espacio-temporales determinados. Teóricamente articula dos enfoques: contienda política 11, que construye una mirada procesual entre actores y estructuras políticas e identifica dos tipos de contienda -contenida y contenciosa o transgresiva-, la primera vincula actores preestablecidos y privilegia escenarios institucionales para canalizar demandas sociales y; la segunda, generalmente implica nuevos actores, usualmente subalternos, recurre principalmente a acciones disruptivas, e innova algunos repertorios. Enfoque construccionista 12 , privilegia la mirada de movimientos sociales y actores colectivos como procesuales, por ello no ve los movimientos sociales como unidad de acción, con identidades cristalizadas, sino como procesos sociales en construcción.

\section{Método}

Es un estudio de caso nacional, reconstruido a partir de: análisis documental, especialmente investigaciones elaboradas previamente por los autores, prensa y documentos del movimiento social; observación participante de los investigadores en diferentes etapas del movimiento social por la salud y; 108 entrevistas semiestructuradas realizadas durante los últimos 10 años por los investigadores a actores claves del campo sanitario y de diferentes regiones: profesionales sectoriales, usuarios y pacientes, población LGTBI, líderes comunitarios, miembros del movimiento social por la salud, sindicalistas del sector, pensionados, indígenas, funcionarios sectoriales, estudiantes del área y población desplazada. Se establecieron cinco períodos, desde finales de los noventa hasta el 2020, definidos por dinámicas de la política sectorial, que condujeron a ciclos de protesta impulsados por organizaciones específicas, permitiendo reconstruir las contiendas -contenida y transgresiva- en salud. La información fue organizada en matrices analíticas y con ella se construyó: línea de tiempo del movimiento social por la salud y de política sanitaria, mapa de actores por periodo, ejes de disputa y repertorios de acción.

Las investigaciones fueron aprobadas en: Acta 045 del 7 de octubre de 2010, Acta 052 de 2 de agosto de 2011, Acta 198 del 30 de octubre de 2018, avaladas por el Comité de Ética de la Facultad 
Nacional de Salud Pública, Universidad de Antioquia y; Acta 001-003-17 de 26 de enero de 2017 del

Comité de Ética de la Facultad de Medicina de la Universidad Nacional de Colombia.

\section{Resultados}

\section{Décadas de reformas y de luchas por la salud}

La construcción durante tres décadas del movimiento social por la salud en Colombia ha sido compleja, y enmarcada en la implantación del SGSSS y la reforma del Estado. Los 1990 parecían un periodo de inflexión, la expedición, en 1991, de una Constitución Política, precedida de un nuevo pacto social y de desmovilización de guerrillas, proclamó a Colombia como un Estado Social de Derecho, realizable priorizando el gasto social; formuló mecanismos de participación y protección de derechos fundamentales, entre ellos la acción de tutela, abriendo la esperanza para avanzar en la democratización y la garantía de derechos sociales. Paralelamente, inició un proceso de reforma del Estado y de política social, orientada por el Consenso de Washington 13, que recomendó -a través del Banco Mundial-, medidas de ajuste estructural 14,15. La tensión entre estos dos proyectos de sociedad en las últimas décadas ha fortalecido la racionalidad mercantil en los sectores del bienestar, debilitando el Estado Social de Derecho; además, las reformas promercado transfieren recursos públicos a agentes privados, convirtiendo la política social en nicho de acumulación de capital, y con ello, en espacio de disputa.

En salud, estas orientaciones concretaron un diseño institucional -pluralismo estructurado-, con un mercado regulado que buscaba combinar las supuestas ventajas del Estado y del mercado 16 . El SGSSS está basado en aseguramiento individual, con dos regímenes: subsidiado para población pobre focalizada, y contributivo para aquellos con capacidad de pago y financiado por trabajadores y empleadores. El aseguramiento es manejado por intermediarios que reciben una prima por afiliado y contratan la prestación de servicios definidos por un plan de beneficios, con instituciones prestadoras, además hay copagos para el acceso. El Estado cumple el papel de "modulador" del sistema y de la "competencia regulada" entre aseguradores y prestadores públicos y privados, además se encarga de los servicios de salud pública 17 .

Los efectos del SGSSS han sido documentados: fortalecimiento de intermediarios privados con concentración de afiliados y creación de red de prestadores propios, con leyes favorables a sus intereses, mientras aseguradores públicos desaparecen 18; hospitales e instituciones públicas de seguridad social transformadas en "empresas" financieramente autosostenibles, con cierres, privatizaciones, despido de trabajadores, precarización laboral, y desmantelamiento sindical; sumado al afán de rentabilidad que impuso barreras de acceso a servicios, incrementando las tutelas en salud. La primacía de servicios asistenciales, en detrimento de la salud pública, provocó caída de las coberturas de vacunación, incremento de las enfermedades inmunoprevenibles; y, en zonas rurales, cierre de puestos y centros de salud y supresión de cargos de promotoras de salud 4,5,6,7. Estos efectos, igual que la indignación y la protesta social, se profundizaron en paralelo con la consolidación de la reforma. El movimiento social por la salud surge y se configura en este contexto, disputando una propuesta alternativa de salud, con gobiernos que, desde los noventa, optan por ajustes legislativos preservando la intermediación y las poderosas aseguradoras privadas 19. La pandemia del COVID-19 plantea retos a ambas partes, ante una realidad abierta al statu quo y/o al cambio.

\section{Transformación del SGSSS y nacimiento del movimiento social por la salud (1998-2001)}

A finales de los 1980 Colombia inició la descentralización, inscrita en acuerdos de negociación con guerrillas desmovilizadas y demandas democráticas de movimientos sociales, así como, procesos de modernización estatal, funcionales, orientados a políticas neoliberales. Estas dos vertientes antagónicas fueron incorporadas en la Constitución Política, y paralelamente, las reformas neoliberales se mantienen como política de Estado, en un contexto paradójico de estabilidad institucional, persistencia de conflicto armado y protesta social. Recogiendo directrices internacionales, el pluralismo estructurado concretó la reforma sanitaria 13,17 . 
En este período, la intermediación financiera de aseguradoras, crisis hospitalaria, despido de trabajadores asociado a reestructuración de hospitales públicos, desmantelamiento sindical, y retroceso de indicadores sanitarios, debido a la priorización de servicios asistenciales rentables y fragmentación de responsabilidades, recursos e información para gestionar la salud pública, marcaron por un lado, el primer ciclo de contienda contenciosa con dos momentos de intensificación de la protesta social en 1999 y 20015 y, por otro lado, la preocupación de un sector de la academia por los cambios mencionados, especialmente la pérdida de fortalezas sanitarias construidas anteriormente 19,20.

Paralelamente, entre 1998-2000, la primera gran crisis económica producto de políticas de ajuste, triplicó el desempleo, además, continuaba avanzando la desregulación estatal de la mano de la creación de nuevas instituciones pro-mercado en los sectores del bienestar, y, empezaba a configurarse una tendencia legislativa -que se mantendrá durante todo el período- hacia el desmonte del Estado Social de Derecho de la Constitución Política 7, con la reforma, en 2001, que disminuyó los recursos transferidos desde la nación a los territorios para la política social. Estos efectos detonaron protestas sociales con participación de sectores de salud, educación y trabajo.

En este contexto, entre 1997-1998, sectores académicos plantean la necesidad de organización, pedagogía y debate crítico, frente al lugar secundario y fragmentado de la salud pública, siendo la base para el nacimiento del movimiento nacional por la salud ${ }^{19}$, posible por la convergencia de cuatro procesos: (i) los efectos de la implantación del nuevo SGSSS ya mencionados; (ii) las tradiciones organizativas, sindicatos del sector salud, asociaciones de usuarios y/o comités de salud, que ante la crisis hospitalaria se articularon para defender hospitales públicos; (iii) la existencia de pensamiento médico-social, afín a la Medicina Social Latinoamericana, que sensibilizó profesionales y académicos del área, frente a los crecientes problemas sociosanitarios; (iv) la alianza, en el año 2000, con la Plataforma Colombiana de Derechos Humanos, Democracia y Desarrollo -convergencia de varias organizaciones- para trabajar una "Campaña por el Derecho a la Salud, abriendo escenarios regionales de articulación entre actores sociales y organizaciones de salud 19,21. Estos procesos propiciaron la realización, en 2001, del I Congreso Nacional por el Derecho a la Salud”, evento simbólico y fundacional del movimiento social por la salud, con más de 100 organizaciones -sindicales, indígenas, campesinas, estudiantiles, académicas-, de todo el país 22, entonces llamado Movimiento Nacional por la Salud y la Seguridad Social (MNSSS).

\section{Construyendo localmente el movimiento social por la salud (2001-2004)}

Período marcado, paradójicamente, por simultaneidad del fortalecimiento local del MNSSS y fractura de la instancia nacional, debido a la participación de algunos integrantes en el gobierno de centroizquierda de Bogotá; en medio de una política social que profundizó el mercado y la desregulación del Estado, y, en salud, el desmantelamiento de instituciones de salud pública y seguridad social, el fortalecimiento de aseguradores privados, y el impulso de un Tratado de Libre Comercio con Estados Unidos 23. El efecto de políticas neoliberales y autoritarias, lideradas por el gobierno de Álvaro Uribe, fue el incremento de la protesta social en 2004 5. Su agenda acogió el enfoque de manejo social del riesgo del Banco Mundial 24, propuso fortalecer la presencia del mercado en la política social y concentrar la intervención del Estado, solo cuando individuo, familia y comunidad no resolvieran en el mercado la provisión de bienes y servicios básicos; creó el Ministerio de Protección Social -fusión de las carteras de salud y trabajo-, disminuyó la financiación de hospitales públicos, y endureció los criterios de focalización para los subsidios de política social y de aseguramiento en salud de población en extrema pobreza 4.

En política de salud, fortaleció aseguradores y prestadores privados, debilitando la seguridad social pública, imponiendo reestructuración y atomización del Instituto de Seguros Sociales y la conversión de hospitales públicos -en crisis crónica- en instituciones financieramente autosostenibles; los procesos de reestructuración y las reformas laborales generaron despidos masivos de trabajadores favoreciendo la competencia, y la expansión no regulada de actores privados que acumulaban poder económico y político para incidir en la política sanitaria 25 , y de paso, fracturaban la acción política de los mayores sindicatos sectoriales, quienes convocaban movilizaciones sociales y la articulación de actores contra las reformas laborales, sociales 4 , además, se oponían a las negociaciones para la firma del Tratado de Libre Comercio con Estados Unidos que favorecía los intereses de la industria 
farmacéutica, profundizando los derechos de propiedad intelectual 26. Paralelamente, el malestar social aumentaba por la exclusión de una franja de población pobre de la focalización, la inequidad por menores contenidos en los planes de beneficio para población subsidiada 4,19 y las barreras de acceso que llevaban a los ciudadanos a recurrir a la judicialización para obtener servicios 27 .

En este conflictivo escenario, el movimiento social por la salud desarrollaba la agenda del I Congreso Nacional por la Salud, con desenlaces heterogéneos; en Bogotá, actores claves del movimiento social por la salud se vincularon al gobierno local alternativo, generando tensiones internas. En contraste, en Medellín, sindicatos, organizaciones comunitarias y estudiantiles en alianza con la academia acompañaban procesos de educación popular; sin embargo, en 2004, una política nacional -que incentivaba las denuncias de presuntos integrantes de grupos armados- atomiza el movimiento social por la salud por la judicialización de líderes sociales de salud. En otras regiones -Magdalena Medio y Cali- la educación popular fue un repertorio de conformación del tejido organizativo. En síntesis, este período representa una fractura que remite a la pregunta sobre participación de los movimientos sociales en los gobiernos; pero también, desde los territorios, evidencia procesos de politización de actores locales y construcción de vínculos de afecto, confianza y solidaridad 19.

\section{Conflicto y reconstrucción de confianza (2004-2010)}

Este período afianza tres procesos que marcan la trayectoria del movimiento social por la salud: la contienda contenida representada en la participación en el debate legislativo, las dinámicas organizativas inscritas en las particularidades locales, y, la vinculación a la contienda contenciosa que alcanza -entre 2007-2010- los picos más altos desde el inicio de la reforma, expresión de la persistencia del malestar social por sus regresivos resultados; la profundización de políticas sociales y sanitarias de impronta mercantil acudiendo a medidas legislativas de excepción, el desmantelamiento del frágil Estado Social de Derecho de la Constitución Política; y la violencia contra sindicalistas del sector salud 4,19.

En el escenario socioeconómico continúan las luchas contra la firma del Tratado de Libre Comercio con Estados Unidos, motivadas por las afectaciones previstas para acceder y producir nacionalmente medicamentos; también, por la decisión de volver permanente la disminución de transferencias nacionales a los territorios, destinadas, por mandato constitucional, a la política social, asunto que detonó protestas en 2007; y, por la respuesta gubernamental represiva 4 . En salud, persisten cierre y privatización de hospitales públicos, despido de trabajadores, y barreras de acceso impuestas por aseguradores y prestadores que ya naturalizaban las violaciones del derecho a la salud y se consolidaba la incidencia de actores privados en la política de salud 4,18. Ante esta situación la Corte Constitucional, partiendo del análisis del incremento de la judicialización en salud, producto de negación de servicios (más de un 70\% en el plan de beneficios 27 ), emitió la Sentencia de Tutela 760 de 2008, ordenando al gobierno asumir medidas regulatorias en salud, y formular una Ley Estatutaria de Salud para definir alcance y contenido del derecho a la salud 28.

A 10 años de la Ley 100, que inició la reforma sectorial, el gobierno nacional promovió, en 2004, una evaluación de la misma; después de una larga contienda legislativa, la Ley 1122 de 200729 representó un ajuste que mantuvo la intermediación, concesiones a aseguradoras privadas, y segmentación del aseguramiento con planes de beneficios diferenciales según posición socioeconómica. Esta coyuntura fractura al movimiento social por la salud, en dos tendencias que se evidenciaron en 2004, durante el II Congreso del Movimiento Nacional por la Salud, realizado en Bogotá: un sector se oponía radicalmente a la participación en debates legislativos, argumentando que no había posibilidad de ganar esa disputa, por las mayorías parlamentarias del gobierno; mientras centrales sindicales, sindicatos de salud, movimiento indígena y sectores del movimiento social vinculados al gobierno alternativo de Bogotá proponían participar con una propuesta legislativa construida desde la sociedad civil 19.

La configuración del movimiento social por la salud estuvo atravesada por los contextos locales 19: la escisión en la instancia nacional; el debilitamiento sindical por despido y violencia antisindical; y, estigmatización y judicialización de líderes en Medellín, que devino en miedo y silenciamiento entre 2004-2006; mientras Cali fortaleció el proceso con formación de líderes de asociaciones de usuarios y veedurías ciudadanas. La oportunidad de articulación y reconstrucción de confianza ocurrió entre 2009-2010 con la declaratoria de "emergencia social" - una medida que otorga poder al ejecutivo para 
legislar en situaciones excepcionales -obviando el trámite en el Congreso-, el gobierno nacional argumentó un colapso inminente del sistema de salud, si no se fortalecían las aseguradoras y las finanzas sectoriales, promulgó leyes para recortar beneficios del plan de salud, restringir la autonomía médica, e incrementar costos a usuarios, quienes deberían asumir con préstamos bancarios y ahorros personales y familiares servicios no incluidos en el plan de beneficios 30 . Estas medidas generaron indignación ciudadana, consenso dentro del movimiento social por la salud, y articulación en la masiva protesta social del 2010 31, logrando que la Corte Constitucional derogara la emergencia social. En este período el movimiento social por la salud gana visibilidad en la esfera pública, transitando del miedo a la recuperación de confianzas, mostrando la potencia de vínculos preexistentes 12 , para configurarse como sujeto colectivo.

\section{Reagrupando las fuerzas (2010-2015)}

La fallida emergencia social y el inicio de la Presidencia de Juan Manuel Santos (2010-2018) inauguran un período de accidentada contienda legislativa en salud que, aunque mantiene la mercantilización y la intermediación con aseguradores, fortalece y visibiliza el movimiento social por la salud, a través de la Alianza Nacional por la Salud (ANSA) y de la Mesa Nacional por el Derecho a la Salud, participando en la contienda contenida con dos proyectos de ley alternativos. Quince años de lucha devienen en la configuración de una identidad anclada en la salud como derecho fundamental, que estructura los procesos organizativos y refleja la madurez política alcanzada, en tanto las iniciativas legislativas oficiales no resuelven la crisis hospitalaria, las barreras de acceso, la primacía del mercado, el uso desbordado de la tutela por la violación sistemática del derecho a la salud, ni el malestar social acumulado

La emergencia social sumó nuevas fuerzas sociales al movimiento social por la salud -gremios médicos rechazaron la injerencia en su autonomía, sectores académicos sustentaron la no resolución de la crisis estructural, directivos alertaron sobre el riesgo de extinción de hospitales por asfixia financiera del sector público, organizaciones de pacientes expresaron incapacidad para pagar servicios costosos, iglesia y partidos políticos pidieron al gobierno derogar los decretos 32-, las multitudinarias marchas de rechazo representaron el segundo mayor pico de protestas, en 2010, desde el inicio de la reforma 5. La derogatoria de la emergencia social por la Corte Constitucional agregó otro elemento de tensión entre poderes ejecutivo y judicial; fue una derrota política al cierre del gobierno uribista, que pretendía favorecer intereses de los aseguradores; y, convocó voluntades para exigir reforma estructural del sistema de salud 33 .

En ese contexto, Santos inicia sus dos períodos de gobierno en agosto de 2010, debía avanzar en la firma del Tratado de Libre Comercio con Estados Unidos y enfrentar la movilización social generada por este y por la crisis del sistema de salud: recurriendo al repertorio legislativo para afrontar problemas estructurales sin tocar sus fundamentos, se expide la Ley 1438 de 201134 consolidando más el mercado sanitario, débilmente regulado por el Estado. Además, la Corte Constitucional había ordenado al ejecutivo, desde 2008, definir el alcance y contenido del derecho a la salud, entonces, el movimiento social por la salud, la ANSA, adelantándose al gobierno, presentaron, en 2012 y 2013, al Congreso de la República, dos proyectos de ley alternativos -Ley Estatutaria de Salud y Ley Ordinaria de Salud 35,36-, para una reforma estructural; dichas iniciativas no lograron avanzar. El gobierno Santos, interpelado por el mandato de la Corte y por la fuerte movilización del movimiento social por la salud, formula, en 2013, la Ley Estatutaria de Salud, pero la Corte condiciona su aprobación a la incorporación, en contravía de la intención gubernamental, entre otras modificaciones sustantivas, de la unificación de los planes de beneficios, la preservación de la tutela para la exigibilidad del derecho a la salud, y la progresividad hacia un contenido integral del derecho a la salud más allá de servicios de asistenciales 32. Aunque el gobierno dilató la aprobación, firmó la Ley Estatutaria de Salud en 2015 37, presionado por sectores ciudadanos, gremiales y académicos, que lo consideraron un logro 38. 


\section{Confluencia para implementar la Ley Estatutaria de Salud y la paz (2016-2019)}

Un periodo inscrito en la fase final de la negociación entre el gobierno nacional y la organización insurgente FARC devino en la firma de los acuerdos de paz, a finales de 2016, en un contexto de polarización nacional entre sectores proclives a la paz y otros opuestos a ella. Para las organizaciones del movimiento social por la salud las demandas apuntaron principalmente a la reglamentación y puesta en marcha de la Ley Estatutaria de Salud y, a la inclusión de la salud en los acuerdos de paz para posibilitar una política y un sistema sanitario garante del derecho a la salud universal.

Así, parte del quehacer del movimiento social por la salud apuntó a acciones contenciosas, de incidencia política y elaboración de propuestas para reglamentar la Ley Estatutaria de Salud, orientadas a mejorar el acceso a servicios de salud, las condiciones laborales de los trabajadores sectoriales y la autonomía profesional, así como la protección y fortalecimiento de la red pública hospitalaria. A pesar de estas demandas del movimiento social por la salud, la Ley Estatutaria de Salud mantuvo el aseguramiento y la intermediación financiera establecidas desde 1993 39, recogiendo la orientación del Ministerio de Salud.

De otro lado, la paz constituyó una bandera central del movimiento social del país en este periodo. En particular, la paz vinculada con la salud era parte de la agenda política del movimiento social por la salud desde sus orígenes. En este sentido, el movimiento social por la salud propuso acciones demandando la firma del acuerdo de paz y la incorporación de la garantía del derecho a la salud, considerándola como requisito sustancial para la paz 40 . Sin embargo, las circunstancias en Colombia cambiaron radicalmente en el segundo semestre del 2018, con la llegada a la presidencia de Iván Duque, quien representa sectores políticos y sociales opuestos a la negociación con la insurgencia.

El gobierno Duque tomó medidas en contra de los acuerdos de paz, y paralelamente, intentó profundizar políticas sociales regresivas, que sectores sociales denominaron "el paquetazo", produciendo una gran agitación social, un paro nacional que inició el 21 de noviembre de 2019 (llamado 21N), y detonó un ciclo de protesta sostenido intensamente hasta mediados de diciembre de 2019. Actores del movimiento social por la salud, principalmente sindicatos, gremios, académicos y estudiantes del sector, se integraron a esa dinámica nacional conformando una instancia denominada "Salud en el Paro", desde la cual lideraron la demanda de salud en el pliego, planteando un pacto social y político por el derecho fundamental a la salud, universal, gratuito, de calidad y sin barreras, que formalizara y dignificara la contratación laboral de los trabajadores sectoriales, entre otros aspectos 41 .

Esta dinámica de movilización de finales del periodo, articuló múltiples sectores y movimiento social integrando jóvenes quienes aportaron a la movilización innovación, con elementos artísticos y culturales, recreando demandas sociales con nuevos lenguajes, poniendo en aprietos políticos al gobierno Duque, pero dos circunstancias generaron su descenso: las festividades de fin de año y la inédita emergencia de la pandemia de COVID-19 en marzo de 2020, llevando a confinamiento generalizado de la población y con efecto desmovilizador, pese a los esfuerzos por mantener virtualmente el paro, representando para el gobierno un salvavidas frente a la enorme presión del estallido social.

\section{Pandemia, intensificación de la lucha de trabajadores sectoriales y nuevos esfuerzos de articulación}

Este periodo -apenas emergente- empieza con la pandemia de COVID-19 y posterior al ciclo de protesta de 2019, liderado por el Comité Nacional de Paro. El primer paciente fue diagnosticado el 6 de marzo de 2020 y el 17 de marzo el gobierno nacional decreto un Estado de Emergencia Económica, Social y Ecológica 42 que otorgó funciones excepcionales al presidente. A partir de esta figura se promulgaron varios decretos orientados, inicialmente, a la declaración de cuarentena; posteriormente, entre otras medidas, flexibilización del confinamiento, apertura de sectores laborales, y algunos subsidios focalizados para las familias más vulnerables. Más adelante, producto de la emergencia, se plantearon medidas como financiar con recursos públicos una aerolínea internacional privada e incluso un proyecto de ley para reformar el actual SGSSS 43 que, profundiza el mercado de salud al mantener el aseguramiento y crear condiciones para favorecer oligopolios.

La preocupación de actores tradicionales del movimiento social por la salud frente al manejo de la pandemia, especialmente por: el aumento del hambre en el país, la deuda histórica de los aseguradores 
con los prestadores -especialmente públicos-, la precariedad laboral en el sector y la falta de equipos de protección, el incremento de las barreras de acceso a atención sanitaria, la ambivalencia del manejo de la pandemia por parte del gobierno, y la baja capacidad de pruebas y rastreo, entre otras cosas, movilizaron a viejos y nuevos actores visibilizando lo que estaba ocurriendo.

Para mediados de julio se empiezan a articular sindicatos tradicionales del sector, nuevos sindicatos médicos creados recientemente, gremios de profesionales de la salud, académicos y actores de outros movimientos como indígenas, campesinos, ambientalistas, organizaciones populares, el comité del paro, entre otros, para construir una plataforma de lucha denominada Pacto Nacional por la Salud y la Vida 44. Esta plataforma demanda actualmente 15 puntos entre ellos: renta básica, investigación y terminación del asesinato de líderes y lideresas sociales, fortalecimiento de la autoridad sanitaria para el manejo del COVID-19, unificación de los recursos de salud con manejo estatal, giro directo de recursos a los prestadores, implementación de un modelo de atención a la pandemia territorializado y orientado por atención primaria en salud, además de una reforma estructural del sistema de salud.

Este proceso organizativo de lucha por la salud demanda hoy al menos tres asuntos: el cuestionamiento al gobierno por el manejo de la pandemia, el archivo del Proyecto de Ley 01045 que busca profundizar la mercantilización sectorial, el acceso universal a vacunas seguras y eficaces, y las denuncias por amenazas de muerte a varios líderes y defensores de derechos humanos integrantes del pacto. Además, el reto de avanzar en la construcción de unidad en dos sentidos: ampliando la plataforma de lucha más allá de la reforma del sistema de salud, articulando las demandas específicas en salud de otros actores sociales, y ampliando el dialogo sectorial con otros sectores sociales para fortalecer la unidad.

En treinta años de lucha, la respuesta del Estado ha sido realizar ajustes legislativos en 2007 29, 2011 34, y 201537 manteniendo la intermediación financiera del aseguramiento, fortaleciendo el mercado y la no resolución de problemas estructurales sanitarios, pese a la Ley Estatutaria de Salud. Paradójicamente, dos Sentencias de la Corte Constitucional 28,32 han sido claves porque instalaron en la mentalidad de los jueces, de la población, y en las luchas del movimiento social, la noción de salud como derecho fundamental autónomo.

\section{Reflexión final}

La pandemia de COVID-19 ha reubicado, en la agenda pública global, la importancia de la salud, las inequidades sociosanitarias, sus determinantes, los sistemas de salud y el valor del trabajo en salud. Esta coyuntura además puso en la escena luchas sociales por la salud en distintos contextos. En España, Francia, Alemania, por ejemplificar, los trabajadores sanitarios han salido a la calle para exigir el fortalecimiento de sistemas públicos sanitarios; en Latinoamérica, Chile incorporó la salud en la agenda de movilización logrando convocar una constituyente; $y$, Brasil fortalece el movimiento sanitarista. La pandemia evidenció lo que las luchas de muchos pueblos han demandado en estos treinta años: la necesidad de sistemas de salud públicos, con trabajo sectorial digno y donde la enfermedad no sea objeto de lucro privado.

Sin embargo, la constitución de un actor colectivo por la salud no parece fácil. Dos preguntas resultan claves: “¿quién es el sujeto político que lucha por la salud?” y, dada la relación entre determinantes sociales de la salud y resultados sanitarios, “`se podrán transformar los resultados de salud cerrando las brechas de inequidad, si el movimiento social por la salud no involucra en sus demandas procesos de determinación social por la salud?”. La situación actual demuestra la profunda interdependencia entre modelo de desarrollo, procesos de jerarquización social, violencias estructurales, inequidades sociosanitarias e impacto sobre la vida de las poblaciones.

La historia del movimiento social por la salud en Colombia, país considerado como modelo a emular por parte de instituciones internacionales 46, muestra que la reforma de mercado en salud generó enorme malestar social y sentimientos de injusticia ${ }^{19}$, movilizando la ciudadanía para constituir un movimiento social 47, sin embargo, dada la heterogeneidad de actores y demandas, ha sido muy difícil consolidar una plataforma de lucha, donde todos se articulen con fuerza para reversar las reformas de mercado. Además, considerando el vínculo entre salud y determinantes, parece insuficiente exigir sistemas universales de salud, se requeriría ampliar la plataforma de lucha, avanzando a un nuevo 
pacto social, que establezca un nuevo modelo de desarrollo, ligado a un sistema de protección social universal, que garantice el conjunto de derechos sociales, incluido el de la salud; pacto como los promovidos en Brasil, donde la lucha por la salud estuvo articulada a la demanda de un nuevo proyecto de sociedad democrático que concluyó con la Constitución Federal de 1988 48, o en Chile, donde la ola de protestas logró pactar la convocatoria a una constituyente que incluya derechos sociales -salud, educación y pensiones-.

\section{Colaboradores}

Y. Borrero-Ramírez aportó en la construcción del proyecto, análisis documental, análisis de la información y escritura del artículo. M. T. Torres-Tovar y M. E. Echeverry-López aportaron en el diseño del proyecto, análisis de la información y escritura del artículo.

\section{Informaciones adicionales}

ORCID: Yadira Borrero-Ramírez (0000-00032559-4637); Mauricio Torres-Tovar (0000-00026232-6706); Maria Esperanza Echeverry-López (0000-0001-9116-5107).

\section{Agradecimientos}

Agradecemos a todos los hombres y mujeres que durante más de 20 años han luchado por el derecho a la salud en Colombia, exponiendo sus vidas en un contexto de conflicto interno armado. Así mismo, agradecemos a nuestras universidades el apoyo para el desarrollo de este tipo de procesos investigativos. Agradecemos el apoyo financiero de la Facultad Nacional de Salud Pública a través del Proyecto INV639-19.

\section{Referencias}

1. Hernández M. La fragmentación de la salud en Colombia y Argentina: una comparación sociopolítica, 1880-1950. Bogotá: Universidad Nacional de Colombia; 2004.

2. Uribe M. La contienda por la reforma del sistema de salud en Colombia (1990-2006) [Tesis de Doctorado]. México DF: Colegio de México; 2009.

3. Torres-Tovar M. Diez años por un movimiento social en salud. Desde Abajo 2011; 18 feb. https://www.desdeabajo.info/colombia/ item/18496-diez-a\%C3\%B1os-por-un-movimiento-social-en-salud*.html.

4. Echeverry-López ME, Borrero-Ramírez YE. Protestas sociales por la salud en Colombia: la lucha por el derecho fundamental a la salud, 1994-2010. Cad Saúde Pública 2015; 31:354-64.

5. Torres-Tovar M, Vega-Romero RR, Luna-García J, Borrero-Ramírez YE, Echeverry-López ME. Luchas por el derecho a la salud en Colombia: vínculos con la salud para todos/as. Saúde Debate 2020; 44:51-63.

6. De Currea V. El derecho a la salud en Colombia, diez años de frustraciones. Bogotá: Ediciones Antropos/Instituto Latinoamericano de Servicios Legales Alternativos; 2003.

7. Echeverry-López ME. Indignación justa: estudios sobre la acción de tutela en salud en Medellín. Medellín: Universidad de Antioquia; 2013.

8. Departamento Administrativo Nacional de Estadística. Estadísticas vitales -EEVV- Defunciones por COVID-19. Boletin Excepcional COVID-19, 2 de marzo al 4 de octubre de 2020. https://www.dane.gov.co/files/investi gaciones/poblacion/defunciones-covid19/bo letin-defunciones-covid-2020-02mar-04oct. pdf (accedido el 02/nov/2020). 
9. Instituto Nacional de Salud. COVD-19 en Colombia. https://www.ins.gov.co/Noticias/ Paginas/Coronavirus.aspx (accedido el 03/ Nov/2020).

10. Pinto MT, Archila M. Idas y venidas. Vueltas y revueltas. Protesta social en Colombia 19581990. 2a Ed. Bogotá DC: Centro de Investigacion y Educacion Popular; 2008.

11. Charles T. Contentious performances. New York: Cambridge University Press; 2008.

12. Melucci A. Acción colectiva, vida cotidiana y democracia. México DF: Colegio de México; 1999.

13. Ahumada C. Reformas del Estado y políticas de salud en Colombia: un enfoque de salud internacional. En: Uribe C, editor. Políticas y servicios sociales para el siglo XXI. Cali: Pontificia Universidad Javeriana; 1999. p. 279-92.

14. Williamson J. El cambio en las políticas económicas de América Latina. México DF: Ediciones Gernika; 1991.

15. Banco Mundial. Invertir en salud. Informe del desarrollo mundial. Washington DC: Banco Mundial; 1993.

16. Hernández M. El concepto de equidad y el debate sobre lo justo en salud. Rev Salud Pública 2008; 10 Suppl 1:72-82.

17. Londoño JL, Frenk J. Structured pluralism: towards a new model for health system reform in Latin America. Health Policy 1997; 41:1-36.

18. Uribe M. ¿Mercado regulado o competencia sin control? Las reformas de la salud en Colombia, 1995-2011. In: Uribe M, editor. Los vaivenes de las políticas sociales en Argentina, Colombia, Chile, México y Uruguay. ¿Neo o posneoliberalismo? México DF: Editorial Porrúa; 2011. p. 33-80.

19. Borrero-Ramírez YE. Lucha por la salud en Colombia. Cali: Pontificia Universidad Javeriana/Medellín: Universidad de Antioquia; 2014.

20. Currea-Lugo V. La salud: de la caridad al negocio sin pasar por el derecho. In: Plataforma Colombiana de Derechos Humanos, Democracia y Desarrollo, editor. La salud está grave. Una visión desde los derechos humanos. Bogotá: Antropos; 2000. p. 189-308.

21. Torres-Tovar M. Experiencia de organización y movilización social: iniciativa por el derecho a la salud 2000-2002. Bogotá: Plataforma Colombiana de Derechos Humanos, Democracia y Desarrollo; 2002.

22. Movimiento Nacional por la Salud y la Seguridad Social. Primer Congreso Nacional por la Salud: conclusiones generales. Bogotá: Ediciones Antropos; 2002.

23. Sarmiento L. Malestar social y política pública. In: Borrero C, editor. El embrujo continúa. Segundo año de gobierno de Álvaro Uribe Vélez. Bogotá: Plataforma Colombiana de Derechos Humanos, Democracia y Desarrollo; 2004. p. 89-97.

24. Holzmann R, Jørgensen S. Manejo social del riesgo: un nuevo marco conceptual para la protección social y más allá. Revista de la Facultad Nacional de Salud Pública 2003; 21:73-106.
25. Crisis en salud ha obligado a más del $50 \%$ de hospitales a endeudarse para solventar gastos. El Espectador 2015; 18 nov. https://www.ele spectador.com/salud/crisis-en-salud-ha-obli gado-a-mas-del-50-de-hospitales-a-endeu darse-para-solventar-gastos-article-600096/.

26. Arévalo LA. Tratados bilaterales de libre comercio y acceso a la salud: el caso colombiano, entre el neoinstitucionalismo y la biopolítica. In: Pontificia Universidad Javeriana; Universidad de San Buenaventura, editores. Globalización, libre comercio y salud. Cali: Ediciones Aurora; 2014. p. 75-96.

27. Defensoría del Pueblo. La tutela y el derecho a la salud 2011. 20 años del uso efectivo de la tutela 1992-2011. https://consultorsalud.com/ wp-content/uploads/2014/10/la_tutela_y_ el_derecho_a_la_salud_2011_defensoria.pdf (accedido el 02/Oct/2020).

28. Corte Constitucional. Sentencia ST n. 760 de 2008. Bogotá: Corte Constitucional; 2008.

29. Congreso de la República de Colombia. Ley 1122 de 2007, por la cual se hacen algunas modificaciones en el Sistema General de Seguridad Social en Salud y se dictan otras disposiciones. Diario Oficial 2007; 9 ene.

30. Presidencia de la República de Colombia. Decreto 4.975 de 2009, por el cual se declara el Estado de Emergencia Social. Diario Oficial 2009; 23 dic.

31. Torres-Tovar M. Ocho años de inseguridad social en salud. Desde Abajo 2010; 3 jun. https:// www.desdeabajo.info/ediciones/item/7067ocho-a\%C3\%B1os-de-inseguridad-social-ensalud.html.

32. Corte Constitucional. Sentencia 313 de 2014. Constitucionalidad del proyecto de Ley Estatutaria por medio del cual se regula el derecho fundamental a la salud y se dictan otras disposiciones. Bogotá: Corte Constitucional; 2014.

33. Movimiento Nacional por la Salud y la Seguridad Social. Colombia. Por una salud con principios. Desde Abajo 2010; 24 mar. https:// www.desdeabajo.info/ediciones/item/6604colombia-por-una-salud-con-principios.html.

34. Congreso de la República de Colombia. Ley 1438 de 2011, por medio de la cual se reforma el sistema general de seguridad social en salud y se dictan otras disposiciones. Diario Oficial 2011; 9 ene.

35. Alianza Nacional por la Salud. Proyecto de Ley Estatutaria de Salud 105 de 2012, por la cual se reglamenta el derecho fundamental a la salud y se dictan otras disposiciones. Bogotá: Gaceta del Congreso; 2012.

36. Alianza Nacional por la Salud. Proyecto de Ley Ordinaria de Salud 233 de 2013, por la cual se crea el Sistema Único Descentralizado de Seguridad Social en Salud. Bogotá: Gaceta del Congreso; 2013.

37. Torres-Tovar M. Los alcances reales de la Ley Estatutaria en Salud. Semanario Virtual Caja de Herramientas 2015; (435). https://viva.org. co/cajavirtual/svc0435/articulo06.html (accedido el 29/Sep/2020). 
38. Mesa Nacional por el Derecho a la Salud. La entrada en vigencia de la Ley Estatutaria en Salud marca un nuevo rumbo a la estructura del sistema de salud. http://www.udea. edu.co/wps/wcm/connect/udea/4e4ccf0e3e3f-43b2-89b1-85ed1d02d711/MNDS+B P1504+La+entrada+en+vigencia+de+la+Le $\mathrm{y}+$ Estatutaria+en+Salud+marca+un+nuevo +rumbo+a+la+estructura+del+sistema+de+ salud.pdf?MOD=AJPERES (accedido el 29/ Sep/2020).

39. Torres-Tovar M. No se partió la historia de la salud en dos. Desde Abajo 2017; 6 abr. https:// www.desdeabajo.info/ediciones/item/31226no-se-partio-la-historia-de-la-salud-en-dos. html.

40. Franco S. La salud y la paz. In: Franco S, editor. Por la salud social. Bogotá: Ediciones Aurora; 2017. p. 289-91

41. Vargas C. Situación de los trabajadores de salud, un año después del $21 \mathrm{~N}$ de 2019. Desde Abajo 2020; 24 nov. https://www.desdeabajo. info/ediciones/item/41081-situacion.delos-trabajadores-desalud-un-ano-despuesdel-21n-de-2019.html.

42. Presidencia de la República. Decreto 417 de 2020, por el cual se declara un "Estado de Emergencia Económica, Social y Ecológica”. Diario Oficial 2020; 17 mar.
43. Congreso de la República. Proyecto de Ley 010, por medio de la cual se dictan disposiciones orientadas a garantizar el Derecho Fundamental a la Salud dentro del Sistema General de Seguridad Social, de conformidad con la ley 1751 de 2015, y la sostenibilidad del Sistema de Salud. Bogotá: Gaceta del Congreso; 2020.

44. Pacto Nacional por la Salud y la Vida. Pacto Nacional de Unidad por la Salud y la Vida de todos los colombianos y colombianas. https:// www.facebook.com/Pacto-Por-La-Vida-Y-LaSalud-Colombiana-632683724041762/pho tos/pcb.632718127371655/63271596070520 5/ (accedido el 07/Ago/2020).

45. Pacto Nacional por la Salud y la Vida. Carta de archivo del PL010. Bogotá: Pacto Nacional por la Salud y la Vida; 2020.

46. World Health Organization. The World Health Report 2000. Health systems: improving performance. Geneva: World Health Organization; 2000.

47. Torres-Tovar M. Lucha social contra la privatización de la salud. Bogotá: Centro de Investigacion y Educacion Popular; 2013.

48. Escorel S. Reviravolta na saúde: origem e articulação do movimento sanitário. Rio de Janeiro: Editora Fiocruz; 2009. 


\section{Abstract}

This national case study of Colombia from 1998 to 2020 used document analysis, interviews, and participant observation to describe the complex process of shaping a social movement that struggles for health. Theoretically, the study adopts a relational perspective that links the focus of the political dispute and the constructivist view to understand the constitution of the social health movement and its relationship to the processes of shaping health policy. The article is structured on framing the consolidation of the neoliberal development model and the structural reform in health during a period of domestic armed struggle and political contention established between the sectors defending the reform and those that reject it and demand a change that guarantees the right to health. The article proceeds to identify and analyze five periods of dispute, each characterized by changes that deepened the market model in health, with the reconfiguration of actors, repertoires for action, and internal tensions that have persisted over time. The conclusions are that this collective actor's identity addresses health as a fundamental human right and that given the heterogeneity of actors and demands, it has been difficult to consolidate an articulated platform of struggle that can reverse the market reforms. In addition, given the link between health and social determinants, it appears insufficient to demand universal health systems, thus requiring the expansion of the platform of struggle.

Right to Health; Community Participation; Social Participation

\section{Resumo}

Essa pesquisa foi um estudo de caso nacional, na Colômbia, entre 1998-2020; que, a partir de análise documental, de entrevistas e de observação participante, descreve o complexo processo de configuração de um movimento social de luta pela saúde. Teoricamente é uma perspectiva relacional, que articulou o enfoque da luta politica e o olhar construtivista, para entender a constituição do movimento social pela saúde e a sua relação com os processos de configuração da política sanitária. $O$ artigo começa contextualizando a consolidação do modelo de desenvolvimento neoliberal e da reforma estrutural em saúde, em pleno conflito armado interno e a luta política que se estabelece entre setores partidários da reforma, frente aos que a rechaçavam e exigiam uma mudança capaz de garantir o direito à saúde. A partir disso, são identificados e analisados cinco períodos de combate, cada um deles caracterizado por transformações que aprofundavam o modelo de mercado na saúde, com a reconfiguração de atores, repertórios de ação e tensões internas que permanecem no tempo. Conclui-se que a identidade de este ator coletivo considera a saúde como direito humano fundamental e que, dada a heterogeneidade de atores $e$ demandas, foi difícil consolidar uma plataforma de luta articulada que consiga reverter as reformas de mercado. Além disso, dado o vínculo da saúde com os determinantes, não parece ser suficiente demandar por sistemas universais de saúde e se faz necessária uma ampliação da plataforma de luta.

Direito à Saúde; Participação da Comunidade; Participação Social
Recibido el 12/Dic/2020

Versión final presentada el 22/Jun/2021

Aprobado el 02/Jul/2021 